\title{
Prontuário Eletrônico: uma ferramenta que pode contribuir para a integração das Redes de Atenção à Saúde
}

\author{
Electronic Medical Record: a tool that can contribute to integration of Health Care \\ Networks
}

João Paulo Pereira Gonçalves', Larice Rodrigues Batista², Larissa Mendes Carvalho³, Michelle Pimenta Oliveira ${ }^{4}$, Kênia Souto Moreira ${ }^{5}$, Maísa Tavares de Souza Leite ${ }^{6}$

1 Graduando em Medicina pela Universidade Estadual de Montes Claros (UNIMONTES) Montes Claros (MG), Brasil. joaopaulopg@gmail.com

${ }^{2}$ Graduanda em Medicina pela Universidade Estadual de Montes Claros (UNIMONTES) -

Montes Claros (MG), Brasil.

larah_lary@hotmail.com

${ }^{3}$ Graduada em Enfermagem pela

Universidade Estadual de Montes Claros

(UNIMONTES) - Montes Claros (MG), Brasil.

laramc18@hotmail.com

${ }^{4}$ Mestre em Cuidado Primário em Saúde pela Universidade Estadual de Montes

Claros (UNIMONTES) - Montes Claros (MG), Brasil. Preceptora do Programa de Educação pelo Trabalho para a Saúde - (PET-Saúde UNIMONTES) - Montes Claros (MG), Brasil. Tutora da Residência Multiprofissional em Saúde da Família para cirurgiões dentistas e enfermeiros da UNIMONTES - Montes Claros (MGSP), Brasil.

mi.auliveira@gmail.com

${ }^{5}$ Especialista em Saúde Coletiva, ênfase em Saúde da Família pela Faculdades Unidas do Norte de Minas (FUNORTE) - Montes Claros (MG) Brasil. Professora contratada da rede SOEBRAS - Associação Educativa do Brast. Enfermeira da Estratégia Saúde da Família - Montes Claros (MG), Brasil. Preceptora do Programa de Educação pelo Trabalho para a Saúde - PET-Saúde (UNIMONTES) - Montes Claros (MG), Brasil.

keniasoutomoreira@hotmail.com

${ }^{6}$ Especialista em Saúde Coletiva, ênfase em Saúde da Família pela Faculdades Unidas do Norte de Minas (FUNORTE) - Montes Claros (MG), Brasil. Professora contratada da rede SOEBRAS - Associação Educativa do Brasil. Enfermeira da Estratégia Saúde da Família - Montes Claros (MG), Brasil. Preceptora do Programa de Educaçăo pelo Trabalho para a Saúde - PET-Saúde (UNIMONTES) - Montes Claros (MG), Brasil.

keniasoutomoreira@hotmail.com
RESUMO Esta pesquisa objetivou investigar a existência e a acessibilidade ao prontuário eletrônico na atenção primária à saúde. Trata-se de estudo quantitativo, descritivo e exploratório, realizado em Montes Claros - Minas Gerais, Brasil. Os dados foram coletados entre nos meses de outubro de 2010 ea março de 2011. A análise foi realizada por meio do Statistical Package for The Social Science PASW ${ }^{\circledR}$ 17.0. Foram entrevistados médicos, enfermeiros e cirurgiõães-dentistas de Equipes de Saúde da Família (ESF). Este trabalho foi aprovado pelo Comitê de Ética em Pesquisa da Universidade Estadual de Montes Claros, parecer consubstanciado no 2011/2010. Os resultados demonstraram a inexistência do prontuário eletrônico.

PALAVRAS CHAVE: Prontuário Eletrônico; Atenção Primária à Saúde; Informática Médica; Saúde da Família; Sistema Único de Saúde.

ABSTRACT This study investigated the existence and accessibility to electronic medical records in health care. This is a quantitative, descriptive and exploratory study, held in Montes Claros Minas Gerais, Brazil. Data were collected from October 2010 to March 2011. The analysis was performed using the Statistical Software Statistical Package for Social Science The PASW ${ }^{\circledR}$ 17.0. We interviewed doctors, nurses and dentists of Family Health Teams. This study was approved by the Ethics Committee in Research of Montes Claros State University, the consolidated $n^{0} .2011 / 2010$. The results showed the lack of electronic medical records.

KEYWORDS: Electronic Medical Records; Primary Health Care; Medical Informatics; Family Health; Unified Health System. 


\section{Introdução}

As Redes de Atenção à Saúde (RAS) são definidas por Mendes (2011) como organizações poliárquicas de um conjunto de serviços de saúde vinculados entre si por missão única e objetivo comum: garantir uma atenção contínua e integrada a determinada população, no tempo e lugar certos, de forma humanizada e com equidade. A estrutura organizacional das RAS é necessária para garantir a efetividade na prestaçáo dos serviços de saúde. Dentre as ferramentas que podem auxiliar essa organização encontram-se os sistemas logísticos, representados pelos cartóes de identificação dos usuários e o prontuário clínico.

Mendes (2010) descreve a constituição das RAS em três elementos: a população, a estrutura operacional e o modelo de atenção à saúde. $\mathrm{O}$ autor ainda considera que os sistemas logísticos constituem componente fundamental para a organização dessa rede, caracterizando soluçôes tecnológicas fortemente ancoradas nas tecnologias de informação, que garantem uma organização racional dos fluxos e contrafluxos de informaçôes, produtos e pessoas nas RAS. Isso permite um sistema eficaz de referência e contrarreferência dos usuários e trocas eficientes de informaçóes ao longo dos pontos de atenção à saúde e dos sistemas de apoio inseridos nas redes.

O prontuário é um documento único constituído de um conjunto de informaçóes, de sinais e de imagens registradas, geradas a partir de fatos, acontecimentos e situações sobre a saúde do paciente e a assistência a ele prestada, de caráter legal, sigiloso e científico, que possibilita a comunicação entre membros de uma equipe multiprofissional (CONSELHO FEDERAL DE MEDICINA, 2002).

Existem outras diversas modalidades de documentos clínicos, dentre eles o Prontuário Familiar, que é definido pela Secretaria de Estado da Saúde do Ceará (2005) como instrumento de integração das informaçôes de saúde dos indivíduos, famílias e comunidades das áreas de atuaçẫo das Equipes de Saúde da Família (ESF).

Entre os tipos de prontuário, encontra-se o eletrônico que, segundo o Institute of Medicine (1997), é um registro eletrônico que reside em um sistema especificamente projetado para apoiar os usuários, fornecendo acesso a um completo conjunto de dados corretos, alertas, sistemas de apoio à decisão e outros recursos, como links para bases de conhecimento médico. Esse tipo de prontuário é uma proposta para atender as demandas dos novos modelos de atenção e de gerenciamento dos serviços de saúde (ORGANIZAÇÃO PANAMERICANA DE SAÚDE, 2003). Sendo assim, este artigo tem como objetivo investigar a existência e a acessibilidade do prontuário eletrônico nos pontos de atenção à saúde de Montes Claros (MG), a partir da atenção primária nas ESF.

\section{Método}

O presente artigo trata-se de uma pesquisa quantitativa, descritiva e de abordagem exploratória, realizada em Montes Claros, município do norte de Minas Gerais, composto por 59 Equipes de Saúde da Família (ESF), 17 Equipes de Agentes Comunitários de Saúde (EACS) e 5 Núcleos de Apoio à Saúde da Família (NASF).

Para a população do estudo, utilizou-se como critério de inclusão a presença de equipe completa e ampliada, constituída por médico, enfermeiro e cirurgiãodentista, devidamente legalizados junto ao Município de Montes Claros, e obteve-se um total de 39 equipes. A amostra foi composta por $20 \mathrm{ESF}$, que representam $33,89 \%$ do total de equipes do município e $51 \%$ do número de equipes completas e ampliadas, devido à indisponibilidade para a entrevista de alguns profissionais das demais equipes por motivo de férias, rescisóes de contrato e outras condiçóes que não permitiram a coleta dos dados simultânea entre todos os integrantes.

A variável avaliada foi a existência do prontuário eletrônico único e se esse poderia ser acessado nos diferentes pontos de atençáo à saúde e nos sistemas de apoio das redes de atenção à saúde de Montes Claros (MG). Para este fim, foi utilizada a questão norteadora retirada do questionário de Avaliação das Redes de Atenção à Saúde (MENDES, 2011): há um prontuário familiar eletrônico único que pode ser acessado nos diferentes pontos de atenção à saúde e nos sistemas de apoio da rede? Esta pergunta foi feita simultaneamente 
aos três profissionais e respondida após discussão prévia entre os mesmos, tendo sido este momento previamente agendado.

A coleta de dados ocorreu entre os meses de outubro de 2010 e março de 2011. Os entrevistadores foram acadêmicos dos cursos de Educação Física, Medicina, Odontologia, Enfermagem e Ciências Biológicas do Programa de Educação pelo Trabalho para a Saúde (PET-Saúde) da Universidade Estadual de Montes Claros (UNIMONTES). A análise dos dados foi realizada empregando-se o programa estatístico Statistical Package for The Social Science PASW ${ }^{\oplus} 17.0$.

A pesquisa foi realizada segundo os princípios éticos da Resolução do Conselho Nacional de Saúde (CNS), $n^{\circ}$ 196/96, No 2011/2010 (CEP/UNIMONTES) e aprovado no Conselho de Ensino Pesquisa e Extensão, resolução do CEPEX no 173 e autorização da Secretaria Municipal de Saúde da Prefeitura de Montes Claros (MG).

\section{Resultados e discussão}

O resultado do estudo aplicado aos profissionais das ESF de Montes Claros (MG) demonstrou que não há implantado no município o sistema de prontuário eletrônico único que poderia ser acessado nos diferentes pontos das RAS e nos sistemas de apoio dessas em Montes Claros (MG).

A cidade estudada possui, atualmente, cerca de 360 mil habitantes e representa o principal polo regional (INSTITUTO BRASILEIRO DE GEOGRAFIA E ESTATÍSTICA, 2010), contando com extenso cadastramento familiar no Sistema de Atenção Básica (SIAB): 62.231 famílias em junho de 2012 (DATASUS, 2012). Os dados justificam a necessidade da implantação de sistemas de gerenciamento de RAS que consigam integrar de forma efetiva as famílias residentes no município em questáo, bem como organizar o atendimento dos usuários do serviço de saúde originários de municípios vizinhos que têm como referência a cidade de Montes Claros (Macrorregiâo). Nesse sentido, um sistema eletrônico estaria bem indicado, devido as suas facilidades de gerenciamento em larga escala.
O que se vivencia em Montes Claros (MG), nas Equipes de Saúde da Família, é a utilização de prontuário centrado na lógica de atendimento individual, que, quando organizado pela família, o que se observa são acúmulos de fichas individuais em um único envelope familiar, apresentando condiçóes precárias, como rasuras, preenchimentos inadequados, incompletos, ilegíveis e com informações sucintas. Essas condições corroboram a falta de segurança, a confiabilidade e a fragilidade no apoio à decisão clínica, prejudicando a atenção/atendimento ao cliente e dificultando a atuação dos profissionais e gestores.

Como as informaçóes contidas no prontuário são a base para a análise da situação de saúde da população adscrita, bem como para a programação das açóes, o controle e a avaliação, além de serem a fonte de alimentação de todos os sistemas de informação do Ministério da Saúde (MINAS GERAIS, 2006), percebemos que o modelo atual utilizado na cidade de Montes Claros (MG) está deficiente perante a proposta dos governos federal e estadual para esse documento.

A Secretaria de Estado de Saúde de Minas Gerais (2006) ressalta as utilidades do prontuário familiar para os usuários, a equipe de saúde e os outros pontos da atenção. São elas:

Aos primeiros, possibilita um atendimento mais seguro e mais eficiente, principalmente quando há necessidade de transferência de setores e/ou de especialistas, uma vez que permite o resgate do histórico dos atendimentos anteriores. As anotaçóes existentes podem dispensar ou simplificar interrogatórios e exames complementares, reduzindo o custo do atendimento e o tempo de permanência no serviço de saúde. Representa, para o usuário, o grande instrumento de defesa, em caso de possíveis prejuízos e de reivindicação de direitos perante o profissional, o serviço de saúde e os poderes públicos.

Para a equipe de saúde, o prontuário familiar deve contribuir para a integração da mesma, possibilitando a coordenação do cuidado, sendo um instrumento de intercomunicação. No ensino e na pesquisa, possibilita o conhecimento dos casos, dos antecedentes, dos contatos e das patologias/condiçóes ou agravos; facilita o estudo de diagnóstico e a avaliação da terapêutica. É 
campo para pesquisa e fonte para os mais diversos dados estatísticos de incidências e prevalências, de morbidade e mortalidade. Além disso, torna possível a verificação e a comparação das diferentes condutas terapêuticas e estabelece uma análise comparativa da eficiência.

Para os outros pontos de atenção, demonstra o padrão de atendimento prestado, facilita a intercomunicação entre os serviços de saúde, possibilitando a continuidade do cuidado para com o usuário. É a base de informaçóes para a interconsulta e o referenciamento, reduz o uso indevido dos equipamentos e dos serviços, evitando a repetição desnecessária de exames e de procedimentos, sendo que permite, a qualquer tempo, um conhecimento exato do tratamento feito e do resultado alcançado.

Vale ressaltar que, de acordo com a literatura, a modalidade eletrônica de prontuário garante que os dados do paciente possam ser compartilhados de forma efetiva e rápida por toda a equipe que atende o paciente, e esse intercâmbio é possível até mesmo em longas distâncias, o que pode também ocorrer nas RAS. Essa ferramenta elimina um problema comum na prática clínica: a ilegibilidade das informaçôes escritas à mão pelos profissionais nos prontuários de papel, fato que pode gerar interpretaçóes errôneas dos dados clínicos e, consequentemente, propedêutica e terapêutica inadequadas para o paciente (PATRÍCIO et al, 2011).

Além disso, no Brasil, são realizadas 360 milhóes de consultas médicas por ano, o que gera um volume crescente de documentos clínicos, tornando inviável o arquivamento dessa quantidade de papel devido aos custos operacionais e à necessidade de grande espaço físico (PATRÍCIO et al, 2011).

Segundo Lavras (2011), a exemplo de outros sistemas de saúde pelo mundo, o Sistema Único de Saúde (SUS) apresenta-se fragmentado, o que gera descontinuidade do atendimento e impossibilita a resposta do mesmo às exigências do quadro de necessidades da saúde. Os autores acreditam, ainda, que a única forma de alcançar a abordagem multiprofissional e o desenvolvimento de práticas de autocuidado é através dos sistemas integrados.

Mendes (2010) concorda com esse autor, acrescentando que os sistemas fragmentados têm sido um desastre sanitário e econômico em todo o mundo. Para ele, esses modelos organizacionais consistem em conjuntos de pontos de atenção à saúde isolados e incomunicados uns com os outros, tendo como consequência a incapacidade de prestarem uma atenção contínua a população. Neste modelo, a atenção primária à saúde não pode exercitar seu papel de centro de comunicação, coordenando o cuidado.

A atenção voltada para a possível fragmentação da atenção à saúde e a preocupação com essa situação não são recentes. Concomitantes a essa observação, surgiram iniciativas de expansão do acesso, para reduzir a segmentação entre os serviços assistenciais e a fragmentação do cuidado à saúde (SILVA, 2011).

Entre as primeiras publicaçóes que abordaram a necessidade de garantir a integridade e a disposição dos sistemas de saúde em redes, está o relatório de Dawson, publicado em 1920, na Inglaterra. Esse documento previa a distribuição dos serviços de saúde conforme as necessidades da população e a interação das ações curativas com as preventivas (SILVA, 2011).

Em 1996, o governo australiano publicou um relatório de avaliação do papel da tecnologia da informação na qualidade da atenção em saúde (AUSTRALIAN HEALTH MINISTERS ADVISORY COUNCIL, 1996). Esse documento concluiu que a informação é item chave para a segurança e a qualidade da atenção à saúde. Esta deve ser garantida de forma correta para os gestores e usuários das redes a fim de produzir mudanças significativas no sistema de saúde. O relatório constatou, também, que as tecnologias de informação na atenção à saúde têm riscos, como a quebra de privacidade. Contudo, melhorias na gerência dos sistemas informatizados podem garantir maior confiabilidade aos mesmos.

No Brasil, desde 1920, com os Centros de Saúde Escola, foram criados vários modelos de sistemas na tentativa de organizar a atenção primária à saúde. As primeiras experiências com Medicina Comunitária na década de 1970, apoiadas pelas universidades, ocorreram em municípios como Montes Claros (MG), Campinas (SP), Niterói (RJ), Londrina (PR), Teresina (PI), São Luis (MA), Cotia (SP), Sete Lagoas (MG), Pelotas (RS) e Joinvile (SC), e, desde então, as estratégias de 
integração evoluíram. Hoje, o sistema vigente em Redes de Atenção à Saúde vem se mostrando efetivo na organização das esferas de gestão, porém, demanda sistemas logísticos evoluídos. Nesse sentido, o prontuário eletrônico poderia ser indicado (LAVRAS, 2011).

As equipes municipais têm papel fundamental na organização do sistema de saúde e precisam estar integradas às esferas estaduais. Para isso, o Conselho Nacional de Secretários de Saúde (CONASS) (CONSELHO NACIONAL DE SECRETÁRIOS DE SAÚDE, 2011) acredita ser imprescindível implementar sistemas de informação potentes, sistemas logísticos e de apoio diagnóstico e terapêutico, além de sistemas de gestáo em Redes de Atenção à Saúde. Sem esses, não é possível uma integração completa entre os diversos pontos de atenção e cuidado. Portanto, é necessária uma atuação consistente da gestão estadual, apoiando técnica e financeiramente as equipes municipais, proporcionando ferramentas para o pleno cuidado.

O sistema informatizado do prontuário clínico permite que as informaçóes sejam guardadas por muito mais tempo, garantindo maior segurança para o paciente e sua família, e facilitando a utilização dos dados em possíveis estudos epidemiológicos. O processamento desses dados possibilita que sejam verificadas as tendências sanitárias em determinada população, despertando previamente a atenção dos profissionais e gestores da saúde para possíveis epidemias, assim provocando medidas imediatas de controle.

As doenças crônicas são um desafio para a atenção primária à saúde, e o registro eletrônico do paciente é o elemento essencial para disparar o provimento de diretrizes na gestão desse tipo de condição (SALTMAN, 2006).

Comparando o prontuário eletrônico ao tradicional de papel, verifica-se que o segundo apresenta algumas desvantagens em relação ao primeiro: não é acessível à distância; só pode estar em um único lugar a cada tempo; sua pesquisa é lenta; é sempre acumulativo - tem-se a história, mas não o resumo até o 'presente' estado; Além disso, os sistemas administrativos - como faturamento e agendamento - requerem esforço manual de integração (UNIVERSIDADE FEDERAL DE SÃO PAULO, 2000).
A utilização do prontuário eletrônico é capaz de aumentar, significativamente, a produtividade e os índices de qualidade e satisfação das pessoas usuárias, o que foi constatado pela Secretaria Municipal da Saúde de Curitiba (PR) (MENDES, 2007).

Silva (2011) destaca a importância do investimento em recursos para a incorporaçáo de tecnologias e a melhoria de acesso dos usuários aos serviços. Segundo o autor, as redes de atenção à saúde têm sido consideradas importante fator de racionalização de gastos e melhor aproveitamento da oferta assistencial disponível. Com essa forma de organização, a economia de escala e escopo torna-se mais evidente.

A modalidade eletrônica ainda permite o armazenamento e a integração dos dados de todo o núcleo familiar, e não apenas do indivíduo, fato esse que não ocorre nas unidades de atenção primária avaliadas (PEREIRA, 2008). De acordo com os dados coletados, observou-se que o prontuário utilizado pelas ESF, além de ser em papel, tem caráter individual, o que contradiz a proposta do Ministério da Saúde, que coloca como atribuição básica de uma Equipe de Saúde da Família a abordagem familiar. Assim, deveria existir, nessas unidades, um documento que abordasse a família de forma holística. Essa atitude é vista como fundamental para que os profissionais de saúde possam correr na esfera psicossocial a fim de prevenir doenças e alteraçóes na funcionalidade familiar, bem como reconhecer seus aspectos evolutivos ao longo do tempo.

Como todo sistema de informação, o prontuário eletrônico tem seus empecilhos, que, muitas vezes, impedem a sua implantação. Dentre eles, podemos citar o alto custo para a implantação dessa ferramenta e a necessidade de treinamento dos profissionais que terão acesso ao mesmo, além do investimento em segurança a fim de garantir o sigilo das informaçóes dos usuários. Entretanto, deve-se verificar o custo-benefício dessa ferramenta, sendo que há evidências de que ela melhora a divisão de trabalho entre diferentes prestadores, reduz a redundância de procedimentos, melhora o diagnóstico e o plano de cuidado (WORLD HEALTH ORGANIZATION, 2001). Dessa forma, os gestores de saúde podem reconhecer as melhorias no funcionamento das RAS nas quais estão inseridos. 
A capacitação dos profissionais inseridos diretamente na utilização das novas ferramentas de comunicação em saúde é necessária para tornar o seu uso eficiente. Esses sistemas necessitam de um nível de informatização adequado para que seus usuários consigam acessar facilmente as informaçóes e gerenciá-las, segundo Saltman (2006). Este autor descreve o modelo ideal, em que os trabalhadores teriam acesso ao computador preferencialmente no ambiente de trabalho imediato, podendo ser fixo ou móvel. Com a modalidade portátil do aparelho, haveria facilidade de atendimento nas visitas domiciliares e no cadastramento das famílias.

O modelo eletrônico de prontuário foi implantado pela prefeitura de Belo Horizonte (MG), onde foram ouvidas as opinióes dos profissionais de saúde sobre essa ferramenta. $\mathrm{Na}$ ocasião, foram encontrados exemplos de resultados positivos que ressaltaram a funcionalidade do prontuário eletrônico do paciente (PEP). Dos 27 entrevistados, 14 profissionais acreditam que a utilização dos protocolos recomendados pelo programa eletrônico ajuda a padronizar o atendimento, e 25 deles citaram a garantia de acesso rápido e a facilidade do compartilhamento de informaçóes (MOURÁO; NEVES, 2007).

Ainda segundo Saltman (2006), infelizmente, muitos profissionais que atuam na atenção à saúde acreditam que a formação atual sobre o uso de computadores pode ser deficitária e pouco atraente. Esse pensamento dificulta a abordagem desses trabalhadores no processo de implantação dos sistemas eletrônicos.

O Serviço Nacional de Saúde no Reino Unido publicou um material contendo elementos indispensáveis para o treinamento em informática da saúde dos seus profissionais (SERVIÇO NACIONAL DE SAÚDE, 1999). Os elementos tratados são: comunicaçáo; gestão do conhecimento; qualidade e gerenciamento de dados; confidencialidade e segurança das informaçóes; usos secundários dos dados e da informaçáo clínica; auditoria clínica e de serviço; sistema de funcionamento clínico e telemedicina; e teleatenção (SALTMAN, 2006). Esses elementos devem ser utilizados no processo de implantação dos sistemas eletrônicos, como o PEP.

A implantação das tecnologias de informação na saúde é tendência mundial. Greiver et al (2011) demonstraram que há experiências bem sucedidas de prontuários mistos em Toronto, no Canadá, onde se utiliza o método eletrônico de registros médicos em alguns aspectos do atendimento, sem que se despreze totalmente o prontuário de papel. O sucesso desse método foi garantido, visto que os grupos de profissionais médicos de família entrevistados nesse estudo canadense não se mostraram dispostos a retornarem à utilização única do registro escrito à mão.

Nesse mesmo estudo, Greiver et al (2011) observaram que alguns participantes classificaram os registros eletrônicos como complexos e inflexíveis, entretanto, atribuíram essas características às interfaces dos modelos utilizados, componentes estes que podem ser aprimorados com o desenvolvimento dos softwares.

Confirmando a eficácia dos modelos eletrônicos de prontuário, médicos entrevistados no trabalho desenvolvido em Toronto (Canadá) acreditavam que a implementação dos registros eletrônicos melhorou a qualidade de seus registros, ressaltando que os prontuários foram mais bem organizados, e que eles foram capazes de encontrar os dados rapidamente.

A acessibilidade descrita no estudo de Greiver et al (2011) foi confirmada por Renée (2010), que comparou a velocidade de busca dos registros através de cronometragem. Este autor constatou que os médicos conseguiram encontrar informaçóes solicitadas com mais facilidade (uma média de 3,2 minutos, em comparação com os 5,9 minutos gastos antes da implementação - $\mathrm{P}$ $\leq 0,01)$.

O objetivo de Renée (2010) foi confrontar a visão do médico e do paciente a respeito da utilização de registros eletrônicos. Nesse estudo, observou-se que a segurança sentida pelo usuário em relação ao profissional, bem como a relação estabelecida entre eles, foi preservada após a implantaçấo da tecnologia em questão. Essa constataçáo confrontou o temor que existia a respeito do prejuízo do relacionamento entre médico e paciente.

Rozenblum et al (2011) avaliaram registros de saúde eletrônicos em diversas regiōes do Canadá e observaram que, em termos de aplicaçóes clínicas, um dos principais benefícios para os pacientes foi a utilização de imagens digitais, bem como a redução de custo.

Nos sistema estadunidense, Kuschnir e Chorny (2010) verificaram a utilização de sistemas integrados 
com planos diferenciados apenas para aqueles que podem pagar, com acesso desigual pela população, não incorporando as questóes de saúde pública e a ação sobre os condicionantes sociais. Segundo a autora, naquele país não se coloca a ideia da regionalização - intrinsecamente derivada dos princípios de universalidade e equidade. Kuschnir e Chorny (2010) atribuíram responsabilidade à gerência dos sistemas nacionais, indicando que a mesma não pode abdicar da função de garantir o direito e a organização das redes regionalizadas, que constituem instrumento para a ampliação do acesso e a diminuição das desigualdades.

Na maioria dos países da América Latina, há uma focalização do acesso à saúde e uma relevância maior é dada aos aspectos de mercado. Especialmente a respeito das duas décadas anteriores a esta, nota-se um padrão de baixo financiamento público, o que corrobora a situação de descentralização observada nesses países (SILVA, 2011). Com o baixo investimento em políticas de gerência em saúde, torna-se difícil investir em sistemas integrados como o prontuário eletrônico para garantir a ampla abrangência dos serviços de saúde à população usuária.

Durante muito tempo, teve-se o entendimento de que as informaçóes contidas no prontuário pertenciam ao médico-assistente ou à instituição. No entanto, hoje, entende-se que é um documento de comunicação entre os profissionais responsáveis pelo cuidado, o usuário e o serviço de saúde. Portanto, não é apenas o registro da anamnese do usuário; trata-se de um instrumento de grande valor, preenchido e utilizado por diversos profissionais de saúde (MINAS GERAIS, 2006). Nesse sentido, a modalidade eletrônica de prontuário é uma ferramenta de grande utilidade para garantir essa integração entre os pontos de atenção.

\section{Conclusão}

O prontuário eletrônico não é utilizado como ferramenta de gestão nas Unidades Básicas de Saúde de Montes Claros (MG). Todavia, é essencial a utilização desse modelo de registro na atenção primária, uma vez que ele proporciona uma maior qualidade ao atendimento e à gestão pública. $\mathrm{O}$ município teria muito a ganhar com a utilização dessa ferramenta: poderia reduzir o número de papéis arquivados, diminuir a ocorrência de erros de conduta; os dados poderiam ser armazenados por mais tempo; evitaria a redundância de procedimentos, tais como exames; aumentaria a produtividade do serviço e a satisfação dos usuários; e facilitaria a intercomunicação nos pontos de atenção.

O prontuário eletrônico apresenta empecilhos para sua utilização, dentre eles: o custo de implantação e a necessidade de capacitação dos profissionais. Contudo, verifica-se que os benefícios gerados superam as dificuldades.

Sendo assim, a implantação dos prontuários eletrônicos em todo o território nacional seria um grande passo em direção à melhoria na qualidade da assistência prestada aos pacientes, por gerar facilidade, agilidade e segurança para os profissionais de saúde. A saúde pública brasileira se tornaria mais completa $\mathrm{e}$ precisa.

\section{Referências}

AUSTRALIAN HEALTH MINISTERS ADVISORY COUNCIL. Taskforce on Quality in Australian Health Care of the Australian Health. Canberra: Department of Health and Aged Care, 1996.

BRASIL. Ministério da Saúde. Departamento de informática em saúde do SUS. DATASUS. Disponível em: <http://www2.datasus. gov.br/DATASUS/index.php?area=0201>. Acesso em: 26 ago. 2012.
CEARÁ. Secretaria de Estado da Saúde. Metodologia de melhoria da qualidade em APS - PROQUALIS. 2. ed. Fortaleza: Secretaria de Estado da Saúde do Ceará, 2005.

CONSELHO FEDERAL DE MEDICINA (Brasil). Resolução no 1.638 de julho de 2002. Define prontuário médico e torna obrigatória a criação da Comissão de Revisão de Prontuários nas instituições de saúde. Brasília: Diário Oficial União, 9 ago. 2002. p. 184-185. 
CONSELHO NACIONAL DE SECRETÁRIOS DE SAÚDE (Brasil). Atenção Primária e Promoção da Saúde. Brasília: CONASS, 2011. 197p. (Coleção Para Entender a Gestão do SUS).

GREIVER, M. et al. Implementation of electronic medical records: effect on the provision of preventive services in a pay-for-performance environment. Canadian family physician Medecin de famille canadien, Mississauga, ON, v. 57, n. 10, p. 381-389, 2011. Disponível em:<http://www.researchgate.net/ publication/51716199_Implementation_of_electronic_medical_ records_effect_on_the_provision_of_preventive_services_in_a_ pay-for-performance_environment>. Acesso em: 16 abr. 2012.

INSTITUTE OF MEDICINE. Division of Health Care Service. Institute of Medicine. National Academy of Science. The computer-based patient record: an essencial technology for heath care. Washington, DC: Institute of Medicine, 1997.

INSTITUTO BRASILEIRO DE GEOGRAFIA E ESTATÍSTICA. Contagem Populacional. Disponível em: <http://www.ibge.gov.br/cidadesat/ painel/painel.php?codmun=314330\#>. Acesso 26 ago. 2012.

KUSCHNIR, R.; CHORNY, A. H. Redes de atenção à saúde: contextualizando o debate. Ciência \& Saúde coletiva, Rio de Janeiro, v. 15, n. 5, ago. 2010. Disponível em: <http://www.scielosp.org/ scielo.php?script=sci_arttext\&pid=S1413-81232010000500006 \&lng=en\&nrm=iso>. Acesso em: 30 ago. 2012.

LAVRAS, C. Atenção primária à saúde e a organização de redes regionais de atenção à saúde no Brasil. Saúde e Sociedade, São Paulo, v. 20, n. 4, p. 867-874, 2011.

MENDES, E. V. A modelagem das redes de atenção à Saúde. Disponível em: <www.saude. es.gov.br/.../A_MODELAGEM_DAS_ REDES_DE_ATENCAO_A_SAUDE.pdf>. Acesso em: 30 abr. 2011.

As redes de atenção à saúde. 2. ed. Rio de Janeiro: OMS/ OPAS, 2011

As redes de atenção à saúde. Ciência \& Saúde Coletiva, Rio de Janeiro, v. 15, n. 5, ago. 2010. Disponível em: <http:// www.scielosp.org/scielo.php?script=sci_arttext\&pid=S1413$81232010000500005 \& \operatorname{lng}=e n \& n r m=i s o>$. Acesso em: 23 ago. 2012.
MINAS GERAIS. Secretaria de Estado da Saúde. Manual do Prontuário da Família. Belo Horizonte: SES, 2006. 210p.

MOURÃO, A. D.; NEVES, J. T. R. Impactos da Implantação do Prontuário Eletrônico do Paciente sobre o Trabalho dos Profissionais de Saúde da Prefeitura Municipal de Belo Horizonte. In: Simpósio de Excelência em Gestão e Tecnologia SEGET 2007, 2007, Resende. Anais... Resende: AEDB, 2007.

ORGANIZAÇÃO PAN-AMERICANA DE SAÚDE; ORGANIZAÇÃO MUNDIAL DE SAÚDE. O Prontuário Eletrônico do paciente na assistência, informação e conhecimento médico. Washington, DC: OPAS/OMS, 2003.

PATRÍCIO, C. M. et al. O prontuário eletrônico do paciente no sistema de saúde brasileiro: uma realidade para os médicos? Scientia Medica, Porto Alegre, v. 21, n. 3, p. 121-131, 2011. Disponível em: <http://revistaseletronicas.pucrs.br/ojs/index. php/scientiamedica/article/ viewFile/8723/6722>. Acesso em: 23 nov. 2011.

ROZENBLUM, R. et al. A qualitative study of Canada's experience with the implementation of electronic health information technology. CMAJ, Bethesda, v. 183, n. 5, p. 281-288, mar. 2011. Disponível em: <http://www.ncbi.nlm.nih.gov/pmc/articles/ PMC3060213/?tool= pubmed>. Acesso em: 16 abr. 2012.

SILVA, S. F. Organização de redes regionalizadas e integradas de atenção à saúde: desafios do Sistema Único de Saúde (Brasil). Ciência \& Saúde coletiva, Rio de Janeiro, v. 16, n. 6, jun. 2011. Disponível em: <http://www.scielo.br/scielo.php?script=sci_ arttext\&pid=S1413-81232011000600014\&lng=en\&nrm=iso $>$. Acesso em: 28 ago. 2012

UNIVERSIDADE FEDERAL DE SÃO PAULO. Faculdade de Medicina. Departamento de Informática em Saúde. Sistemas de informação em saúde. São Paulo: Faculdade de Medicina da UNIFESP, 2000.

WORLD HEALTH ORGANIZATION. European Office for Integrated Health Care Services. Workshop on Integrated Care. Barcelona: WHO Integrated Care Meeting, 2001.

Recebido para publicação em em Abril/2012

Versão final em em Janeiro/2013

Conflito de interesse: Não houve.

Suporte financeiro: Inexistente. 